\title{
Mechanism and Pathway of Nonylphenol Degradation in Aqueous Solution by Dielectric Barrier Discharge
}

\author{
Rongjie YI, Haijun YU, Qi ZHANG, Liu YANG and Chengwu YI ${ }^{1}$ \\ School of Environment and Safety Engineering, Jiangsu University, Zhenjiang 212013, \\ China
}

\begin{abstract}
Nonylphenol (NP) is a typical environmental endocrine disruptor with low concentration and high toxicity, $\mu \mathrm{g} / \mathrm{L}$ level of NP is enough to interfere with the sex differentiation of many aquatic organisms and affect the population dynamic balance. The mechanism and pathway of NP degradation in aqueous solution by strong ionization dielectric barrier discharge were studied. The results showed that the degradation rate of NP can reach $95.2 \%$ in 60 min when the flow rate of $\mathrm{O}_{3}$ is $5 \mathrm{~L} / \mathrm{min}$ and the input voltage is $3.8 \mathrm{kV}$. Based on the electron spin resonance (ESR) results and the detection of $\mathrm{O}_{3}$, a large amount of hydroxyl radical $(\cdot \mathrm{OH})$ and $\mathrm{O}_{3}$ were verified. In addition, the ability of DBD method to degrade NP was also tested. The intermediate products were analyzed by liquid chromatography-mass spectrometry (LC-MS), and the degradation pathway was speculated.
\end{abstract}

Keywords. Dielectric barrier discharge, nonylphenol, ozone, hydroxyl radical

\section{Introduction}

Nonylphenol (NP) is the main degradation product of nonylphenol polyoxyethylene ethers (NPEOs), which widely exists in the natural environment. At present, the NP concentration of natural water in China is about $30.05-288.75 \mathrm{ng} / \mathrm{L}$ [1], and that of some watersheds is as high as $33200 \mathrm{ng} / \mathrm{L}$ [2], which is far higher than the national standard $(6.6 \mu \mathrm{g} / \mathrm{L})$. NP structure is similar to estradiol, so it can simulate natural estrogen, affect sex differentiation and reproduction of organisms, and destroy liver, kidney and gonad tissue of organisms [3]. Studies have found that NP can induce apoptosis, autophagy and necrosis of cells, to a certain extent, it can induce the production of cancer cells [4]. At present, researchers have detected the presence of NP in decidua and early embryos of pregnant women, and found that it has obvious maternal transfer, which indicates that NP pollution can't be ignored [5].

Due to the high toxicity and stable structure of NP, it is difficult for microorganisms to degrade it $[6,7]$. However, physical and chemical methods are mostly the transfer of pollutants and can't completed degrade NP. In this case, advanced oxidation technology (AOPs) has gradually become the focus of research on NP degradation. Fenton methods with catalysts have become the new favorite of AOPs,

${ }^{1}$ Corresponding Author, Chengwu YI, School of Environment and Safety Engineering, Jiangsu University, Zhenjiang 212013, China; Email: yichengwu0943@163.com. 
the new methods are indeed effective in the degradation of nonylphenol [8], but the problems of its service life and recovery are still difficult to solve [9]. In contrast, low temperature plasma technology does not need reagents or catalysts, and the degradation process is highly efficient and non-selective, which shows great prospects in the treatment of such refractory organic compounds.

Dielectric barrier discharge (DBD) is one of the most representative low temperature plasma technologies. Because of its simple reaction structure, stable discharge state and large amount of active substances, DBD has become one of the most widely used discharge forms. Compared with other plasma discharge methods $[10,11]$, DBD can produce uniform and stable streamer discharge [12], and the ionization degree of particles is stronger, and the energy of active particles produced by DBD is much higher than that of similar discharge methods [13]. In this experiment, the strong ionization discharge method is used to further optimize the conventional DBD, improve its energy efficiency, expand the processing scale, and achieve a processing scale of $1.8 \mathrm{t} / \mathrm{h}$.

In this study, the performance of DBD device was explored, and NP was taken as the main research object. The degradation ability of DBD system to NP was determined by UV-Vis, TOC and other methods. The intermediate products and final products were analyzed by LC-MS and other analysis methods, and the possible degradation pathways of NP degradation were proposed.

\section{Material and Methods}

\subsection{Experimental Materials}

Nonylphenol (NP, 99\%) was purchased from Aladdin Industrial Corporation Ltd. Acetonitrile and methanol were purchased from China Pharmaceutical Co., Ltd.

\subsection{Experimental Apparatus}

The experimental device mainly includes dielectric barrier discharge (DBD) reactor, high-voltage and high-frequency power, gas-liquid circulation system and electric monitoring system (figure 1). The power frequency voltage of $220 \mathrm{~V} / 50 \mathrm{~Hz}$ is converted into high frequency voltage of $2.5 \sim 4 \mathrm{kV}, 15 \mathrm{kHz} \sim 23 \mathrm{kHz}$ by high-frequency high-voltage power supply, which is input to the DBD generator. The raw material $\mathrm{O}_{2}$ and $\mathrm{H}_{2} \mathrm{O}$ are ionized and excited to produce a large number of active particles, such as $\mathrm{O}_{3}, \cdot \mathrm{OH}, \mathrm{O}_{2}{ }^{+}, \mathrm{O}_{2}{ }^{-}$, etc. In order to achieve the purpose of purifying organic pollutants, active substances mainly composed of $\mathrm{O}_{3}$ and $\cdot \mathrm{OH}$ are formed to oxidize and degrade pollutants in water. 

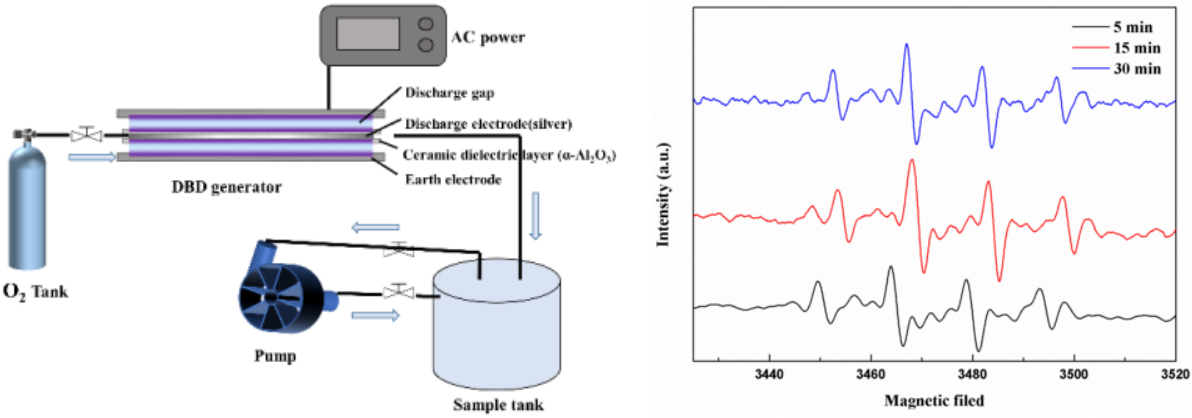

Figure 1. The flow-process diagram of water treatment Figure 2. ESR spectrum of free radicals collected systems of strong ionization discharge. after different reaction times.

\subsection{Analytical Methods}

The concentration and the absorption wavelength of NP was measured by UV-visible spectrophotometer (UV22450, Shimadzu, Japan). The mineralization of the NP was detected using a TOC analyzer (TOC-VCPH/CPN, Shimadzu Corporation, Japan). High concentration ultraviolet dissolved water ozone analyser (CL-7685, B\&C electzanice, Italy) and an electron paramagnetic resonance spectrometer (ESR) (Bruker, MicroESR, Germany) were used to measure the concentrations of $\mathrm{O}_{3}$ and $\cdot \mathrm{OH}$ generated during the process. The intermediate byproducts of NP were measured by high-performance liquid chromatography mass spectrometry (LC/MS/MS) (7890B-5977B, Agilent, USA).

\section{Results and Discussion}

\subsection{Determination of Major Reactive Species in the Reaction Process}

The type and quantity of active particles present are important parameters for evaluating the treatment effect of a large-volume strong ionization DBD water treatment system. DMPO was used as a spin-trapping reagent for the hydroxyl groups, and the free radicals in the strong ionization discharge DBD system were measured using an ESR spectrometer. In the ESR spectrometer (figure 2), the 1:2:2:1 quadruple signal characteristic of DMPO- $\cdot \mathrm{OH}$ adducts appeared. The concentration of $\cdot \mathrm{OH}$ in the $\mathrm{DBD}$ reactor was $0.32 \mathrm{mmol} / \mathrm{L}$ (figure 3). Based on the signal in the ESR spectrometer, it can be seen that the concentration of the hydroxyl group increases with the reaction time. It is confirmed that $\cdot \mathrm{OH}$ plays an important role in the degradation of quinoline. In this experiment, the concentration of $\mathrm{O}_{3}$ in the outlet of DBD system and then dissolved in water was determined under the voltage of $2.1 \mathrm{kV} 2.8 \mathrm{kV}, 3.0 \mathrm{kV}, 3.3 \mathrm{kV}$ and $3.8 \mathrm{kV}$, respectively. As illustrated in figure 4, with the increase of the input voltage, the content of $\mathrm{O}_{3}$ produced by the DBD system also increases. When the applied voltage reaches $3.8 \mathrm{kV}$, the amount of $\mathrm{O}_{3}$ produced reaches $56.9 \mathrm{mg} / \mathrm{L}$ and the dissolved amount in water reaches $14.22 \mathrm{mg} / \mathrm{L}$. 


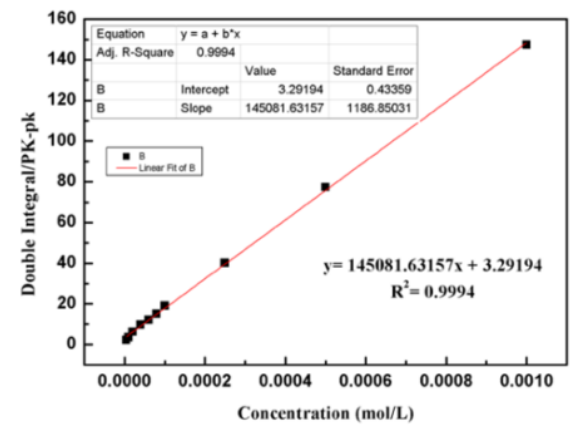

Figure 3. A plot of calibration curve between different concentration and absorbance of DMPO.

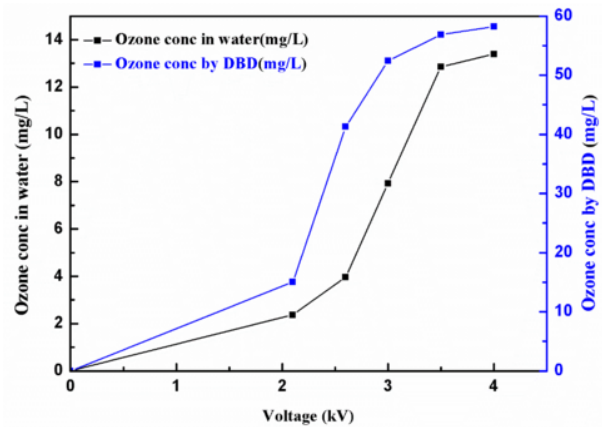

Figure 4. The concentration of $\mathrm{O}_{3}$ in water and in DBD.

\subsection{Analysis of UV-Vis and TOC Results}

As can be seen from figure 5, the maximum absorption wavelength of NP is at $274 \mathrm{~nm}$. The degradation rate of NP has reached about $95 \%$ in $30 \mathrm{~min}$, and the characteristic peak of NP can hardly be scanned in $60 \mathrm{~min}$. Besides, the peak intensity decreased significantly with increasing reaction time. This can be explained by the fact that the carbon ring in the NP structure was gradually disassembled under the conditions of the DBD, generating a large number of small molecular organic compounds.

As shown in figure 6 , the TOC decreased from 15.6 to $8.3 \mathrm{mg} / \mathrm{L}$ in $60 \mathrm{~min}$ using the DBD system, and its degradation efficiency reached $46.7 \%$, while the NP removal efficiency reached $95.2 \%$ under the same experimental conditions. It can be seen that many small molecule intermediates are not completely degraded into $\mathrm{H}_{2} \mathrm{O}$ and $\mathrm{CO}_{2}$.

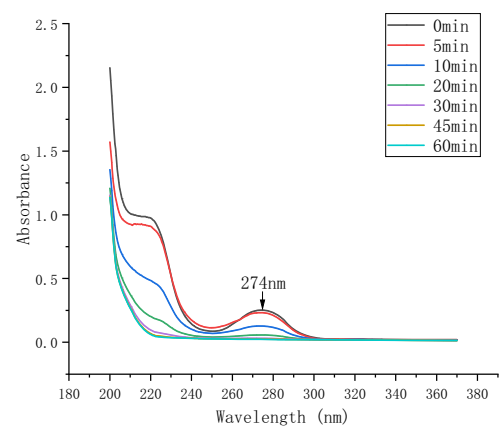

Figure 5. UV-vis analysis of NP solutions of 0-60 min during NP degradation.

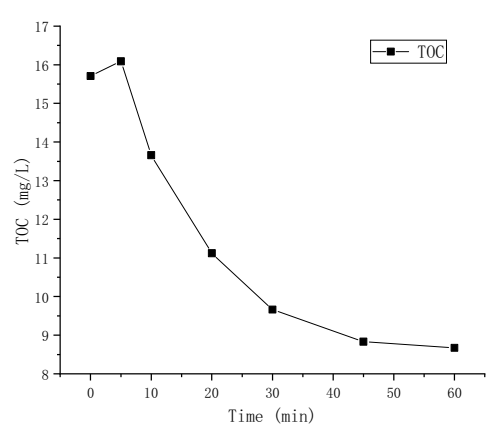

Figure 6. The TOC change in degradation process of NP.

\subsection{Analysis of LC-MS Results}

In order to further explore the mechanism of the OTC degradation via DBD system, LC-MS was used to determine the intermediate by-products formed during the NP degradation process. Due to the fact that the simulated wastewater itself is made by dissolving NP in tap water and contains certain impurities, and the treatment system is 
an open system, and some instruments and equipment cannot be manipulated in the detection process, it is necessary to remove the impurity compounds in the degradation products in the mass spectrometry analysis.

According to the intermediate products, it is speculated that the degradation process of NP by the dielectric barrier discharge device is as shown in figure 7 below. It mainly goes through the following two ways: a part of the $\mathrm{C}-\mathrm{C}$ bond on the alkyl chain of NP is attacked and broken by $\mathrm{O}_{3}$, free radicals and other active particles, and the broken bond combines with hydrogen ions in water to produce alkylphenols. The same process continues to make the alkyl chain finally fall off and become phenol. The other part of NP was attacked by $\cdot \mathrm{OH}$, the $\mathrm{C}-\mathrm{H}$ bond of alkyl was broken, the hydrogen ion was replaced by hydroxyl to form nonylphenol hydroxyl compound, and then the $\mathrm{C}-\mathrm{C}$ bond was broken and returned to the previous way. In addition, some literatures mentioned that benzoquinones would be produced in the process of NP degradation [14], but the presence of benzoquinones was not detected in this experiment. It is speculated that benzoquinone itself is not stable enough and it is easy to be converted into hydroquinone. The presence of hydroquinone was also found in LC-MS results, which verified this point. Finally, under the action of $\mathrm{O}_{3}$ and $\cdot \mathrm{OH}$, the benzene ring was opened and finally oxidized to small molecule carboxylic acids and $\mathrm{CO}_{2}$. According to the detected mass spectra and the previous literature, the possible intermediate products are inferred as shown in table 1.

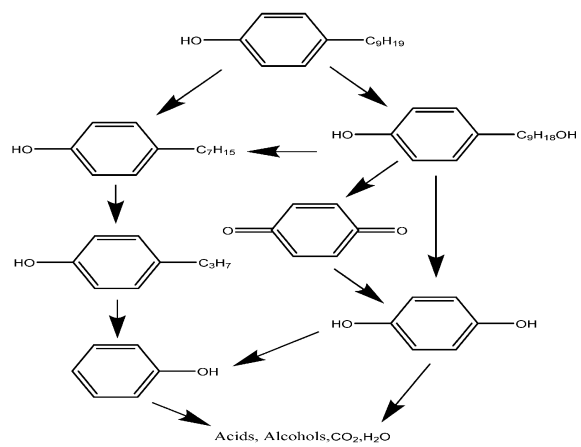

Figure 7. Proposed reaction pathway for the degradation of NP by DBD.

Table 1. NP intermediates via LC-MS analysis.

\begin{tabular}{lll}
\hline No. & Chemical formula & Molecular weight $(\mathbf{m} / \mathbf{z})$ \\
\hline 1 & $\mathrm{C}_{15} \mathrm{H}_{24} \mathrm{O}$ & 221.01 \\
2 & $\mathrm{C}_{15} \mathrm{H}_{24} \mathrm{O}_{2}$ & 239.05 \\
3 & $\mathrm{C}_{13} \mathrm{H}_{20} \mathrm{O}$ & 192.90 \\
4 & $\mathrm{C}_{11} \mathrm{H}_{16} \mathrm{O}$ & 165.01 \\
5 & $\mathrm{C}_{9} \mathrm{H}_{12} \mathrm{O}$ & 137.00 \\
6 & $\mathrm{C}_{6} \mathrm{H}_{6} \mathrm{O}_{2}$ & 112.05 \\
7 & $\mathrm{C}_{6} \mathrm{H}_{6} \mathrm{O}$ & 95 \\
\hline
\end{tabular}




\section{Conclusions}

In this paper, the performance of dielectric barrier discharge (DBD) device was studied. The ozone and $\cdot \mathrm{OH}$ produced in the process of wastewater degradation by strong ionization discharge were qualitatively and quantitatively analyzed. With the increase of voltage, the concentration of $\mathrm{O}_{3}$ gradually increased and then tended to be stable. The change of $\cdot \mathrm{OH}$ and other free radicals was basically the same as that of $\mathrm{O}_{3}$. After that, the mechanism and pathway of NP degradation by DBD were studied. It was found that the removal efficiency of NP in wastewater by DBD was $95.2 \%$, but the removal rate of TOC was only $46.7 \%$, indicating that many intermediate products could not be completely decomposed into $\mathrm{CO}_{2}$ and $\mathrm{H}_{2} \mathrm{O}$. The intermediate products of NP degradation were detected by LC-MS. according to the detection results, it was speculated that the process of NP degradation was relatively simple. In the early stage, the main reason was the breaking of alkyl chain. NP removed different amounts of alkyl to form alkylphenols, and $\cdot \mathrm{OH}$ attacked $\mathrm{C}-\mathrm{H}$ bond to form nonylphenol hydroxylate. Phenol and hydroquinone were the important intermediate products, In the later stage, the benzene ring was attacked by $\mathrm{O}_{3}$ and $\cdot \mathrm{OH}$, and phenol and hydroquinone were oxidized to small molecule carboxylic acids and $\mathrm{CO}_{2}$.

\section{Acknowledgements}

Thanks to the National Natural Science Foundation of China (32071521), the Postgraduate Research \& Practice Innovation Program of Jiangsu Province (No. KYCX18_2272), the Priority Academic Program Development of Jiangsu Higher Education Institutions (PAPD) and Jiangsu Collaborative Innovation Center of Technology and Material of Water Treatment for their support of this work.

\section{References}

[1] Jin X W, Wang Y Y, Jin W and Rao K F 2014 Ecological risk of nonylphenol in China surface waters based on reproductive fitness Environmental Science \& Technology 48 1256-62.

[2] Pei G, Li Z, Gibson M and Gao H 2014 Ecological risk assessment of nonylphenol in coastal waters of China based on species sensitivity distribution model Chemosphere 104 113-119.

[3] Iman S, Mohammad R K, Milad E and Boshra T 2020 Disruptive effects of nonylphenol on reproductive hormones, antioxidant enzymes, and histology of liver, kidney and gonads in Caspian trout smolts Comparative Biochemistry and Physiology Part C 232.

[4] Sun C H, Chou J C, Chao K P, Chang H C, Lieu F K and Wang P S 2019 17 $\alpha$ Ethynylestradiol and 4-nonylphenol stimulate lung adenocarcinoma cell production in xenoestrogenic way Chemosphere 218.

[5] Chen M, Fan Z L, Zhao F R, Gao F M, Mu D, Zhou Y Y, Shen H and Hu J Y 2016 Occurrence and maternal transfer of chlorinated bisphenol a and nonylphenol in pregnant women and their matching embryos Environmental Science \& Technology 50 (2) 970-7.

[6] Ruixia H 2009 Structure-biodegradability relationship of nonylphenol isomers during biological wastewater treatment process Chemosphere 75 (8) 987-994.

[7] Cui K, Yi H, Zhou Z J, Zhuo Q F, Bing Y X, Guo Q W and Xu Z C 2014 Fenton oxidation kinetics and intermediates of nonylphenol ethoxylates Environmental Engineering Science 31 (5) 217-224.

[8] Bechambi O, Najjar W and Sayadi S 2016 The nonylphenol degradation under UV irradiation in the presence of $\mathrm{Ag}-\mathrm{ZnO}$ nanorods: Effect of parameters and degradation pathway Journal of the Taiwan Institute of Chemical Engineers 60 496-501.

[9] Tang C J, Huang X R, Wang H Y, Shi H J and Zhao G H 2020 Mechanism investigation on the enhanced photocatalytic oxidation of nonylphenol on hydrophobic $\mathrm{TiO}_{2}$ nanotubes Journal of 
Hazardous Materials 382.

[10] Lei W, Jiang X Z and Liu Y J 2007 Efficient degradation of nitrobenzene induced by glow discharge plasma in aqueous solution Plasma Chemistry and Plasma Processing 27 (4) 504-515.

[11] Du C M, Zhang L L, Wang J, Zhang C R, Li H X and Xiong Y 2010 Degradation of acid orange 7 by gliding arc discharge plasma in combination with advanced Fenton catalysis Plasma Chemistry and Plasma Processing 30 (6) 855-871.

[12] Yi R J, Guo H, Wang H J, Du D L, Zhang Q and Yi C W 2021 Multiple production of highly active particles for oxytetracycline degradation in a large volume strong ionization dielectric barrier discharge system: Performance and degradation pathways Separation and Purification Technology 274119103.

[13] Yi R J, Yi C W, Du D L, Zhang Q, Yu H J and Yang L 2021 Research on quinoline degradation in drinking water by a large volume strong ionization dielectric barrier discharge reaction system Plasma Science and Technology https://doi.org/10.1088/2058-6272/abffa9.

[14] Ali R R, Mehdi S, Amir S, Nader S, Somaye B and Yaser V 2020 Sono-catalytic activation of persulfate by nZVI-reduced graphene oxide for degradation of nonylphenol in aqueous solution: Process optimization, synergistic effect and degradation pathway Journal of Environmental Chemical Engineering 8 (5). 